\title{
Geografías de la movilidad académica internacional: globalización y discursos de internacionalización
}

César-Augusto Ferrari-Martínez

\section{RESUMEN}

Este artículo presenta una revisión de literatura sobre geografías de la movilidad académica internacional, analizando sistemáticamente 61 trabajos publicados sobre el tema. Utiliza como categorías de análisis tres constructos geográficos operantes en imaginarios de internacionalización: globalización, límite/frontera y escala geográfica. Los resultados son presentados y discutidos a partir de tres grupos: una perspectiva optimista acerca de la movilidad académica, una perspectiva crítica que denuncia y demuestra las contradicciones del proceso y un acercamiento post moderno que pone en duda los supuestos fijos que operan en entendimientos de los estudiantes en movimiento. Se concluye que los enunciados de escala promocionados por discursos de movilidad académica internacional actúan produciendo espacios asimétricos en las geopolíticas del conocimiento global.

Palabras clave: movilidad académica internacional, globalización, estudiantes internacionales. 


\section{Geografias da mobilidade acadêmica internacional: globalização e discursos de internacionalização}

\section{RESUMO}

Este artigo apresenta uma revisão de literatura sobre geografias da mobilidade acadêmica internacional, analisando sistematicamente 61 trabalhos publicados sobre o tema. Utiliza como categorias de análise três constructos geográficos operantes em imaginários de internacionalização: globalização, limite/ fronteira e escala geográfica. Os resultados são presentados e discutidos a partir de três grupos; uma perspectiva otimista sobre a mobilidade acadêmica, uma perspectiva crítica que denuncia e demonstra as contradições do processo e uma aproximação pós-moderna que põe em dúvida os supostos fixos que operam em entendimentos dos estudantes em movimento. Se conclui que os enunciados de escala proporcionados por discursos de mobilidade acadêmica internacional atuam produzindo espaços assimétricos nas geopolíticas do conhecimento global.

Palavras chave: mobilidade acadêmica internacional, globalização, estudantes internacionais.

\section{Geographies of International Academic Mobility: Globalization and Discourses of Internationalization}

\section{ABSTRACT}

This article presents a review of the literature about geographies of international academic mobility, with a systematical analysis of 61 published works on the subject. It uses as categories of analysis three geographic constructs that are particularly useful for studies about imaginaries of internationalization: globalization, limit/boundary and geographic scale. The results are presented and discussed gathering them in three different groups; an optimistic perspective on academic mobility, a critical perspective that denounces and demonstrates the contradictions of the process, and a postmodern approach that questions the fixed assumptions that operate in the understandings of students in motion. The author concludes that the scale statements promoted by discourses of international academic mobility act by producing asymmetric spaces in the geopolitics of global knowledge.

Key words: international academic mobility, globalization, international students. 


\section{Introducción}

Desde la caída del muro de Berlín en 1989, los discursos espaciales dominantes inducen a la idea de un mundo abierto, sin fronteras y de libre circulación (Rosière, 2015). En la educación superior, estos discursos se articulan nombrados como internacionalización, es decir, prácticas institucionales que insisten en la necesidad de adoptar ciertos modos de hacer comunes en la gestión, la educación y la investigación (Sidhu et al., 2016). Entre otras prácticas, la movilidad académica internacional es considerada como uno de los elementos que representa la cultura de internacionalización en las universidades (Matus, 2016). A consecuencia de la difusión de la movilidad como estrategia de internacionalización, entre 2003 y 2013, hubo un crecimiento de más del 100\% de los estudiantes matriculados fuera de su país de origen (UNESCO, 2016). Del mismo modo, crecieron los estudios acerca de esas experiencias internacionales y de cómo actúan produciendo espacios, instituciones y sujetos (Dolby, 2008).

La educación internacional como campo viene siendo abordada a partir de diversos ejes y desde distintas disciplinas. Más allá de tratarse de un tema estrictamente pedagógico, investigaciones recientes reportan sus impactos en políticas de fronteras (Walker, 2013), en economía de Estado (Dennis, 2016), en desarrollo urbano (Collins, 2014), entre otros. La geografía de la educación se dedica a complejizar supuestos operantes en las ideas de espacio asumidas por la educación internacional. Es importante profundizar las dimensiones políticas de la movilidad, ya que se plantea que los movimientos de los estudiantes no son sólo consecuencia de una cartografía política, sino que la producen directamente (Rizvi, 2011; Waters, 2016). Por ejemplo, si se comprende espacios como sinónimo de distancia, la globalización, con sus avances técnicos, los estaría disminuyendo. No obstante, al comprender el espacio como un producto de las relaciones sociales, la globalización - y, por ende, la movilidad académica- sería un catalizador importante en la producción de espacio (Massey, 2008).

De esa manera, en esta revisión se busca mapear cómo se entiende la globalización en la literatura sobre movilidad académica internacional y, también, de qué manera la idea de lo global articula la producción de espacios entre estudiantes internacionales. En primer lugar, se busca contestar la pregunta ¿cuáles son los discursos que promueven la internacionalización en los estudios de movilidad académica internacional? Luego, se indagan las nociones de escala, de fronteras, de lo global, de lo internacional, y se averigua cómo esas conceptualizaciones producen y son producidas por los sujetos que se mueven para estudiar. Se entiende que la educación internacional necesita exponer y profundizar supuestos que operan invisibles, problematizando la narrativa de la globalización como un evento natural y entendiéndola como un proyecto económico y político (Massey, 2008).

\section{Metodología}

\section{Búsqueda y selección de artículos}

A partir del interés creciente de las universidades en atraer estudiantes (Gao, 2014; Ye, 2015), la investigación en educación internacional está altamente mediada por terminología utilizada también en el área del mercado. Por ello, se justifica la necesidad de discutir críticamente las palabras clave articuladas en la búsqueda y en la selección de artículos científicos, pues son necesarias ciertas desambiguaciones para obtener distinciones más precisas en los resultados. La selección de la literatura está planteada por la necesidad de aportes investigativos que: I) indaguen sobre los estudiantes internacionales de educación superior, II) se enfoquen en la movilidad académica como proceso o fenómeno, y III) estén contextualizados en un proceso de globalización.

Conforme apunta Lyotard (1984), el vocabulario no es sólo una representación, sino también un juego de lenguaje. Vale decir que las formas de nombrar 
son instancias enunciativas, prácticas discursivas que se asocian para reproducir un poder (Castro, 2011). En este caso, la forma de denominar la globalización y la educación internacional constituyen metanarrativas (Lyotard, 1984) de una geopolítica internacional. Si, por un lado, el término bordeless education refuerza la idea de una globalidad unitaria, el uso de cross-border education reconoce las fronteras como elementos regulatorios del espacio. La mercantilización en red y los acercamientos económicos al fenómeno de la movilidad acuñaron el término transnational education como el área que investiga los hubs educacionales, es decir, espacios destinados a la atracción ostensiva de estudiantes internacionales. Se percibió, además, el uso de nomenclaturas específicas a ciertos espacios con tradición en movilidad, como overseas education, en el Reino Unido, y offshore education, en Australia. En ese sentido, se tomó la decisión de no identificar la educación internacional utilizando términos específicos para incluir en la búsqueda la diversidad de designaciones geográficas. Lo mismo ocurre con la ambigüedad terminológica en la categorización de "estudiante internacional". Aunque la literatura, muchas veces, no establezca esa distinción, se optó por emplear el término international students como palabra clave, teniendo en cuenta una modalidad específica de migración que se diferencia por su naturaleza y características del extranjero estudiante. En la tabla 1 se presentan las palabras clave utilizadas y sus respectivas justificaciones.

Tabla 1. Palabras clave utilizadas en la búsqueda y sus justificaciones

\begin{tabular}{|l|l|}
\hline \multicolumn{1}{|c|}{ Palabra clave } & \multicolumn{1}{c|}{ Justificación } \\
\hline "International students" & $\begin{array}{l}\text { La elección de identificar la educación internacional con la palabra estudiantes se justifica no sólo en el hecho de poner } \\
\text { énfasis en sus experiencias y procesos, sino también en evitar la expresión "study abroad" que en las búsquedas se presenta } \\
\text { muy asociada a factores de elección y a educación como mercado. }\end{array}$ \\
\hline $\begin{array}{l}\text { "Higher education" or "Tertiary } \\
\text { education" or "Post-secondary } \\
\text { education" or "Third level }\end{array}$ & $\begin{array}{l}\text { De acuerdo con lo mencionado anteriormente, se buscaron las principales formas de nombrar la educación superior } \\
\text { de manera de minimizar sesgos geográficos en la búsqueda. Con ello también se buscó excluir resultados que hicieran } \\
\text { referencia a otras formas de movilidad, como las de educación básica y secundaria o específicas para el aprendizaje de una } \\
\text { segunda lengua. Esa decisión enfatiza la universidad como una institución fundamental en la comprensión de la movilidad. }\end{array}$ \\
\hline "Mobil*" or "Flow" or "Flux" & $\begin{array}{l}\text { Se parte de la necesidad de comprender que el movimiento es fundamental para la producción del espacio (Massey, } \\
\text { 2008). Por lo tanto, se establece como prioridad el análisis de los artículos que no sólo se centraron en políticas de } \\
\text { internacionalización, sino que hicieron un análisis de uno o más flujos de movilidad académica de estudiantes. }\end{array}$ \\
\hline "Global*" & $\begin{array}{l}\text { Este filtro restringe los resultados a las investigaciones que presentan alguna relación entre el proceso de globalización y } \\
\text { la movilidad de estudiantes y es fundamental en las búsquedas para entender los desplazamientos en procesos políticos } \\
\text { espaciales más amplios (globali*ation, globalized, global competences). }\end{array}$ \\
\hline
\end{tabular}

Fuente: organizada por el autor (2017)

La búsqueda de las palabras clave se cumplió en dos etapas. En la primera, se utilizó la base de datos Web of Science, ${ }^{1}$ considerada un medio que concentra artículos de alta relevancia para la comunidad científica internacional. En marzo de 2017, se seleccionaron 54 artículos después de filtrar por criterios de idioma (inglés, portugués, español) y de año (investigaciones publicadas a partir de 2010). Se limitó a artículos que incluyeran todos los filtros mencionados en la tabla 1. En una segunda etapa, durante el mismo mes y año, se amplió la selección a la producción regional latinoamericana con el objetivo de identificar acercamientos locales al respecto del problema. Ese movimiento también responde a una brecha en la producción académica, que es la existente entre los hablantes de portugués y de español, conforme alerta Kritz (2015). Por otra parte, quiere reducir, por lo menos en el nivel latinoamericano, el sesgo

\footnotetext{
${ }^{1}<$ webofknowledge.com $>$.
} 
provocado por una literatura mayoritariamente escrita en inglés. En la base de datos Scielo, ${ }^{2}$ la búsqueda se realizó restringida a la palabra clave international students. Dos artículos fueron descartados por abordar temáticas de aprendizaje de segunda lengua. La búsqueda dio como resultado un total de 61 trabajos.

\section{Categorías de análisis}

Uno de los principales roles de la teoría sobre globalización en las últimas dos décadas fue desnaturalizarla, indicándola como estrategia de reproducción de ciertos modos de interactuar. Para muchos autores (Collins, 2014; Findlay et al., 2012; Kirkegaard y Nat-George, 2016; Santos, 2000), esos modos reproducen y/o sostienen relaciones de poder. Por lo tanto, se hace necesario, además de comprender la globalización a modo de un proceso político articulado y disperso, cuestionar las ideas operantes de Estado-nación como una estructura estable. En este análisis se busca comprender el rol de la nación y cómo ese espacio explica el aprendizaje de estudiantes en movimiento.

El análisis de los artículos se desarrolló teniendo en cuenta de qué forma los discursos de globalización producen, atribuyen calidad y jerarquizan estudiantes, instituciones y espacios. Por lo tanto, se establecieron las categorías de globalización, frontera y escala, por entender que la idea de internacional, como un adjetivo de la movilidad académica, transita por nociones de Estado-nación y los supuestos que se atribuyen a él. Para codificar los resultados se delimitaron marcadores de producción, es decir, subcategorías o términos que se relacionan directamente con los objetivos propuestos, de acuerdo con lo descrito en la tabla 2.

Tabla 2. Categorías de análisis y marcadores de producción

\begin{tabular}{|l|l|}
\hline \multicolumn{1}{|c|}{ Palabra clave } & \multicolumn{1}{c|}{ Justificación } \\
\hline Globalización & Transnacionalización, geopolítica, flujos, redes, macro y microrregiones, mercantilización, conectividad, neoliberalismo. \\
\hline Frontera & Límite, nacional, internacional, cruce, acceso, extranjero, inmigrante, inclusión, exclusión, normalidad, diferencia. \\
\hline Escala & $\begin{array}{l}\text { Inmersión, interculturalidad, global, local, campus internacionalizado, embodiment (corporeización/materialidad), } \\
\text { experiencia, clasificación, jerarquía, excelencia, calidad. }\end{array}$ \\
\hline
\end{tabular}

Fuente: organizada por el autor (2017).

\section{Resultados}

Los artículos fueron agrupados bajo tres perspectivas teóricas distintas: una denominada optimista sobre la movilidad académica; una crítica sobre los contextos que producen la movilidad; y una poscrítica que busca deconstruir los supuestos fundacionales sobre los cuales operan ideas de movilidad académica. En esta sección se presentan los resultados obtenidos a partir de las investigaciones de estos tres grupos, que fueron definidos por la similitud teórica con la que tratan las categorías analizadas.

\section{Globalización}

Los artículos que presentan una discusión optimista acerca de la globalización la comprenden como un proceso naturalizado de integración de los espacios globales. Las investigaciones reportan resultados que demuestran que las prácticas de internacionalización promueven el desarrollo de competencias culturales importantes para vivir la contemporaneidad. Entre las conclusiones del abordaje optimista están: el impacto de la pasantía en un desarrollo multicultural (Brodin, 2010; Lo-Philip et al., 2015); el impacto de la

\footnotetext{
${ }^{2}$ Scientific Electronic Library Online <www.scielo.org>.
} 
internacionalización del currículo para integrar estudiantes extranjeros y locales (Soria y Troisi, 2014), y el desarrollo de una consciencia ambiental como consecuencia de procesos de internacionalización (Wynveen y Tarrant, 2012). Es decir, un estudiante sometido a experiencias interculturales tiene propensión a tornarse más flexible ante la diversidad, a esta característica algunos autores la denominan de ciudadanía global (Brodin, 2010; Higgins y Brady, 2016; Killick, 2011; Wynveen et al., 2012).

Baldassar y Mckenzie (2016) resaltan, sin embargo, que esas competencias sólo son desarrolladas cuando hay un plan de trabajo que involucre a los estudiantes para lo que van a aprender en las pasantías, además de un trabajo de evaluación y síntesis después de su retorno. Doyle et al. (2010) llegan a un resultado semejante y complementan indicando también que las experiencias internacionales pueden aportar siempre que los estudiantes estén involucrados en una diversidad de actividades. Otros resultados ponen en duda ese aprendizaje, pues indican que la investigación hecha a partir de los reportes de los propios estudiantes internacionales - sea cualitativa o cuantitativa - promueve que se reproduzcan los discursos que se esperan de ellos. En otras palabras, hay una repetición de los discursos de internacionalización de las universidades y de agencias de intercambios (Salisbury et al., 2011; Forsey et al., 2012).

Un segundo grupo de artículos adopta una postura más crítica sobre la globalización. Por ejemplo, su idea como integración y apertura es cuestionada por el análisis de macroflujos, es decir, movimientos analizados a partir de datos globales de desplazamiento. Estas investigaciones ponen en evidencia que los estudiantes no eligen libremente, pues están condicionados por estructuras sociales y políticas (Barnett $e t$ al., 2015; Chankseliani, 2016). Shields (2014) analiza datos sobre los movimientos globales de estudiantes y sintetiza que los flujos están cada vez más concentrados, corroborando las conclusiones de Perkins y Neumayer (2014), que encontraron enlaces coloniales entre las decisiones de destinación de estudiantes internacionales. Ye (2015) encuentra que, además, esos flujos priorizan universidades de habla inglesa en un proceso que Geddie (2015, traducción propia) conceptualiza como "conectividad selectiva" y que consiste en la prioridad geopolítica que se le atribuye a destinos comprendidos como de excelencia. Los estudios de Findlay (2011; Findlay et al., 2012) indican que la movilidad académica, además de sus atributos pedagógicos, sirve como reproducción de una clase de élite global, demostrando que la decisión de estudiar en otro país no es más que otra estrategia de reproducción de capital cultural.

Los discursos de valoración de la educación internacional han facilitado el estudio de la relación entre globalización y movilidad académica internacional. Uno de los ejes que se despliega de ese cuerpo de literatura son las investigaciones acerca de la educación transnacional. Los estudios conducidos se concentran en puntos de la región de Asia oriental, entre los que destacan Singapur (Collins et al., 2014; Weiss y Ford, 2011; Ye, 2015; Ziguras y Pham, 2014), Malasia (Abd Aziz y Abdullah, 2014; Weiss y Ford, 2011) y Hong Kong (Gao, 2014; Waters y Leung, 2016; Ziguras y Pham, 2014), y dedican especial interés a cómo algunos espacios se constituyen en nodos en una red global de producción de conocimiento. La transnacionalización se basa en un discurso de calidad de educación internacional y, de acuerdo con Ye (2015), se convierte en una práctica que promueve diferenciación entre estudiantes, es decir, estableciendo posiciones desde las cuales se ejercen privilegios La universidad transnacional se establece a partir de marcas de universidades reconocidas en ciertos espacios del globo. Conscientes de que las universidades de países periféricos no disfrutan de la misma legitimidad que las de los países centrales, esas instituciones pasan a comprar o aceptar marcas de universidades reconocidas (Healey y Michael, 2014).

Ese proceso, al que Geddie (2015, traducción propia) define como "mercantilización en red", provoca la erosión de los límites entre conocimiento y 
producción (Rizvi, 2011). La transnacionalización consolida el proceso de globalización de la educación superior (Pherali, 2012), ubicando a esas universidades en los nodos de grandes flujos de capital (Shields, 2014a). Por lo tanto, la transnacionalización vendría a ser el uso de la educación superior para ofrecerle capital humano a la transnacionalización de la economía en un proceso denominado "economía del conocimiento" (Chiang, 2012; Geddie, 2015; Pherali, 2012; Shields, 2014a). Los resultados de esa línea reportan que las relaciones entre las universidades sede y las universidades sucursales son asimétricas (Chiang, 2012; Healey y Michael, 2014) y reproducen un modelo meritocrático de educación (Waters y Leung, 2016).

La influencia de perspectivas poscríticas, por su parte, tornan más complejo el hecho de que la globalización neoliberal produce subjetividad en los estudiantes internacionales. A ese proceso Cefai (2014, traducción propia) lo denomina de "mercantilización del deseo", entendiendo que se genera a través de discursos de calidad y excelencia en la internacionalización de la educación superior. Es por eso que, para Rizvi (2011), los estudiantes en movilidad no son apenas producto de la globalización, sino que la producen reiterativamente, pues ejercen un rol de agente de esos discursos. Para el autor (Rizvi, 2011) los imaginarios neoliberales reproducen un discurso de mercado que lleva a creer que Estados-nación, instituciones y personas están globalmente conectados. Sidhu y Dall'Alba (2012) señalan que la idea neoliberal de calidad pasa a operar para clasificar no sólo mercados, sino también instituciones, espacios y sujetos.

\section{Fronteras}

La frontera promueve una distinción entre espacios y en ese concepto está basada la idea de internacional en la que se sostienen buena parte de los discursos en movilidad. Los artículos escritos desde una perspectiva optimista de movilidad académica entienden la frontera como el lugar de cruce hacia un espacio diferente y en eso se justifica el aprendizaje. Esa diferencia se establece a partir de nuevos juegos y configuraciones culturales y sociales con las cuales el estudiante negocia y, así, promueve su aprendizaje, como concluye Anderson (2014).

Sin embargo, entre los partidarios de la corriente crítica, la frontera es interpretada como un aparato regulatorio importante para la comprensión de la movilidad. Walker (2013) demuestra que, históricamente, Reino Unido ha utilizado la legislación sobre inmigración para, por un lado, desincentivar el ingreso de extranjeros y, por otro, para estimular la entrada de estudiantes internacionales con recursos. Geddie (2015) muestra que la movilidad internacional de estudiantes es un foco de atención del sector público, reproduciendo las configuraciones geopolíticas de la movilidad académica. Las contradicciones entre esa naturaleza porosa de frontera se hacen más evidentes en el estudio de King et al. (2013), que demuestra que los discursos reproducen la posición paradojal del estudiante internacional: si, por un lado, el mundo post 11 de septiembre lo anuncia como amenaza, por otro, las políticas de internacionalización promueven un esfuerzo para atraerlo. Esa dualidad se convierte en un dilema en constante movimiento para los formuladores de políticas públicas, como sugiere Tannock (2013). En la literatura crítica también se describen otras formas de producir la frontera. Uno de los elementos que constituyen un marco regulatorio para los estudiantes internacionales es el idioma. Eso porque el inglés adoptado por las universidades internacionalizadas puede representar un mecanismo discriminatorio entre académicos y estudiantes (Sterzuk, 2014). Por otra parte, en caso de regiones multilingües, el idioma puede producir fronteras internas, por ejemplo, académicos que hablan inglés en relación con aquellos que hablan otros idiomas nativos (Sibomana, 2016).

Entre los académicos poscríticos se examina de qué forma se vive la frontera y se materializa en los cuerpos de los estudiantes internacionales, entendiendo 
que es como una experiencia subjetiva que no puede entenderse de una forma homogénea (Doughty y Murray, 2016; Kirkegaard y Nat-George, 2016; Schartner, 2016). En todos estos trabajos se desafia la idea de conocimiento como objeto independiente al sujeto que lo produce, y la idea de que globalización y flujo libre corresponden a una estrategia para hacer prevalecer una epistemología occidental (Sidhu y Dall'Alba, 2012). En este sentido, Van Oorshot (2013) argumenta que, si las fronteras son vividas desde los cuerpos, también se materializan situaciones geopolíticas asimétricas en contextos de internacionalización. En otras palabras, se sienten en la carne las jerarquías y las subordinaciones espaciales.

\section{Escala}

La escala es entendida genéricamente en ciencias sociales como la dimensión espacial de un evento. La literatura optimista ofrece tres conceptos en los que se encuentran presentes acercamientos de escala: interculturalidad, inmersión y campus internacionalizado. El estudio de Maringe y Sing (2014), por ejemplo, presenta la interculturalidad como un desafio para la educación superior, argumentando que los contextos educacionales de las naciones de origen de los estudiantes valorizan de forma diferente las prácticas cotidianas realizadas en clases. Es decir, plantea la cultura como un atributo colectivo entre personas de una misma nacionalidad. Esa diferencia la plantean también Soria y Troisi (2014), que hacen una distinción cultural entre estudiantes domésticos e internacionales. La idea de inmersión, en ese mismo sentido, está planteada en el estudio de Doyle et al. (2016) y se justifica en las ventajas del estudiante que quiere aprender inglés en Nueva Zelanda, pues estaría inmerso en una comunidad hablante del idioma.

Algunos trabajos de orientación crítica investigan cómo nociones de calidad son atribuidas a territorios más que a experiencias. En este sentido, el Estadonación y la universidad articulan sus discursos para atribuirle excelencia a los espacios (Gong y Huybers,
2015). Uno de los aparatos utilizados para difundir instituciones consideradas de excelencia académica son los rankings universitarios, como lo demuestran Furukawa et al. (2013) al presentar los impactos que ejercen esos dispositivos en la toma de decisión por parte de los estudiantes. Dichos autores también abordan resultados que demuestran que los estudiantes se postulan a universidades reconocidas para poder insertarse en una red de excelencia global. En otras palabras, las instituciones internacionalizadas permiten acceder a escalas globales en las trayectorias académicas y profesionales. Como consecuencia de ese proceso, Kim (2016) reporta prácticas de diferenciación entre académicos coreanos que obtuvieron sus diplomas de grado en Estados Unidos y los que lo hicieron en Corea del Sur, enfatizando que los diplomas estadounidenses son altamente valorados en la academia coreana.

Otro punto que emerge de las discusiones críticas es la relación entre escala global y local, denunciando nociones de representatividad en medios universitarios. La idea de campus internacional como un espacio que congrega a todas las culturas es criticada por Li y Lowe (2016), pues, conforme argumenta también Pasztor (2015), son conformados por élites nacionales. Lee y Sehoole (2015), por su parte, identificaron un juego geopolítico en la movilidad, prestándole atención no sólo a los grandes flujos globales, sino también a las escalas regionales. Dicho de otra manera, además de las grandes regiones de origen y destinación, hay flujos regionales específicos. Los trabajos de Shields (2014b) y Chankseliani (2016) también identifican los flujos a escalas regionales.

Las investigaciones poscríticas, en ese sentido, aportan análisis sobre cómo los discursos de internacionalización son productores de escala. Sidhu y Dall'Alba (2012) critican una posición binaria en los estudios de movilidad entre lo nacional y lo extranjero, pues esa perspectiva comprende la nación como un espacio estable y homogéneo. Doerr (2014) presenta dos críticas: la movilidad como promotora de 
discursos productores de escala, una vez que centra en la nación las ideas de valores y culturas; en segundo lugar, la noción de inmersión como aprendizaje de otra cultura, pues los datos producidos a partir de la inmersión apuntan que la identidad nacional se negocia cuando se está en movilidad. De acuerdo con Rizvi (2011), los supuestos atribuidos a la nación son repasados a los sujetos, y la cultura como un atributo fijo niega los procesos de devenires que viven los estudiantes cuando negocian sus identidades culturales. Douglass (2015) describe de qué forma las instituciones de educación superior consideradas internacionalizadas postulan una idea de diversidad cultural cómo en un desfile de juegos olímpicos. Es decir, cada estudiante representa una parte del globo, una nación, una cultura, una raza.

\section{Discusión}

Los aportes levantados en los resultados permiten distinguir claramente las corrientes teóricas que influyen en cada perspectiva. El acercamiento optimista está marcado por una comprensión de la movilidad como resultado de un mundo abierto y diverso culturalmente. En esta perspectiva, predominan nociones frágiles de frontera y supuestos de que los estudiantes son sujetos que disponen de todos los recursos necesarios para elegir racionalmente. Prevalecen investigaciones de tenor cognitivista, donde el aprendizaje es medido u observado en contextos curriculares y la cultura es comprendida como un desafío pedagógico para las universidades. La noción operante de escala, por ejemplo, puede producir aportes esencialistas en cuanto a los sujetos, como la descripción de un "modo asiático de aprender", de Maringe y Sing (2014). Las investigaciones tienden a apuntar soluciones institucionales, pedagógicas y administrativas, pero no cuestionan un modelo en específico de globalización que genere esos contextos. Lo global es asumido como realidad, no como proceso.

Los estudios críticos, por su parte, presentan flujos condicionados en los cuales los estudiantes están sometidos a procesos sociopolíticos macroestructurales. Las tres perspectivas más presentes son: los estudios posmarxistas, la teoría del capital de Bourdieu y las teorías de sistema-mundo. Las investigaciones abarcan la comprensión de geopolíticas globales, procesos económicos y condicionantes de clase. Teóricos críticos se vienen dedicando a denunciar lógicas de mercado en las dinámicas de educación superior. Power y Frandji (2010) presentan ejemplos de cómo las prácticas de mercado naturalizan una estructura jerárquica entre las instituciones, reproduciendo inequidades entre estudiantes. Como resultado de lo anterior, las universidades también pasan a operar como instituciones corporativas, objetivando estándares de calidad definidos por una cultura de auditoría (Apple, 2005). Las teorías críticas cuestionan la globalización, pues la entienden como una estrategia político-económica que genera severas inequidades.

Los estudios poscríticos, a su vez, se dedican a explorar, a partir de datos menores (Koro-Ljungberg, 2015, traducción propia), los contextos de producción y negociación de los conocimientos. Los avances con relación a los estudios críticos están en incorporar perspectivas feministas post estructurales, haciendo que los conceptos operantes no sean fijos, sino situados a partir de una materialidad vivida. Por eso se cuestionan los supuestos fundacionales a partir de los cuales se profieren los discursos de movilidad académica internacional. Además de ello, partiendo de aportes post modernos, se realiza la crítica de que lo neoliberal no sólo articula un modelo económico, sino que produce subjetividad en los estudiantes. Esta perspectiva aporta orientaciones potencialmente políticas para entender los cuerpos y, más específicamente, se dedica a complejizar la idea de deseo.

Por la misma razón, se destacan los estudios que profundizan nociones de escala y analizan de qué forma los espacios son clasificados y jerarquizados por los discursos. Para futuras investigaciones, el tema de cómo los estudiantes performativizan esos espacios, como lo entiende Nelson (1999), se presenta como 
potente en la explicación de la producción de subjetividades en movilidad. La idea de escala como un discurso de materialización de poder en el espacio también es un tópico no explorado. Estudios de cartografias posrepresentacionales ofrecen la posibilidad de comprender la movilidad a partir de estrategias de gobierno (Brown y Knopp, 2006) y en la relación entre espacio, escala y epistemologías (Kaiser y Nikiforova, 2008). La desigualdad entre espacios de excelencia y otros espacios (The West and the Rest) puede ser indagada no sólo a partir de la relación dominado/dominante, sino también por los mecanismos por los que circulan y se sostienen esas estrategias discursivas. Por ejemplo, es notable la escasez de estudios que aborden la cuestión de cómo se produce la diferencia a partir de los cuerpos de los estudiantes en movimiento. Cuestiones como raza, género y clase social son tomados como criterios fijos y no como procesos negociados y vividos por los estudiantes.

En ese sentido, se proponen miradas más críticas de los conceptos fundacionales que operan en la movilidad académica. La interculturalidad, por ejemplo, de la forma como es comprendida en investigaciones que asocian linealmente nación, cultura y sujeto, sostiene una idea de territorio como contenedor (Matus, 2016), naturalizando los procesos sociales. Así, los campus internacionales son entendidos como simulacros del mundo, donde cada sujeto representa su continente/nación/raza/cultura. Esas nociones fijas de espacio plantean a la nación como un espacio estable y a los sujetos como metasíntesis de sus naciones de origen. Finalmente, se sugiere explorar la idea de fronteras vividas, considerando que los atributos geopolíticos puedan ser instancias donde se vive la materialización de los discursos nacionales. Doreen Massey (2013) ofrece un aporte importante para la investigación en internacionalización con el concepto de geometrías del poder para identificar la forma como cada local vive de manera distinta las consecuencias de los discursos de globalización.

\section{Conclusiones}

Entre el grupo de artículos analizados, se pueden identificar dos grandes marcos críticos responsables por reorientar y resituar a los estudios de movilidad académica internacional. Primeramente, la inserción de discusiones políticas y económicas críticas al proceso de globalización, permitió lanzar cuestionamientos hacía a la perspectiva celebratoria de la movilidad académica internacional, entendiendo los juegos de poder en que ella se produce. Posteriormente, con los aportes de las teorías feministas, que estas relaciones de poder se gatillan de manera diferenciada en cada cuerpo y trayectoria. Eso demuestra la importancia y necesidad de promover una agenda de investigación en educación internacional que desarrolle teóricamente a la globalización y que investigue sus desdoblamientos sociales.

La producción de discursos sobre movilidad académica internacional encuentra en los imaginarios neoliberales de globalización (Rizvi, 2011) el soporte que necesita para reproducirse. Esta forma de razonar basada en la universidad como una institución competitiva actúa produciendo un espacio que es altamente clasificado y jerarquizado. Por diversos aparatos, la inequidad entre las instituciones y naciones se reproducen en una cartografía de límites, generando un discurso normativo acerca de la calidad educativa. Esos discursos no se restringen sólo a los problemas sugeridos por las teorías críticas en cuanto a la posibilidad o imposibilidad de moverse para estudiar, sino que se traducen también en comprender otras formas de movilidad que no instauren en los territorios una valoración previa. Esa valoración hace uso del deseo de los estudiantes para mantener un status quo de ciertos modelos de pensamiento, sosteniendo como globales prácticas impuestas por sistemas culturales hegemónicos. 


\section{Referencias}

Abd Aziz, M. I. y D. Abdullah (2014), "Finding the next "wave" in internationalisation of higher education: focus on Malaysia", Asia Pacific Education Review, 15(3), pp. 493-502.

Anderson, V. (2014), “World-travelling': a framework for re-thinking teaching and learning in internationalised higher education", en Higher Education, 68(5), pp. 637652.

Apple, M. (2005), "Education, markets, and an audit culture", Critical Quarterly, 47(1-2), pp. 11-29.

Baldassar, L. y L. Mckenzie (2016), "Beyond 'just being there': teaching internationalization at home in two qualitative methods units", Teaching Sociology, 44(2), pp. 84-95.

Barnett, G., M. Lee, K. Jiang y H. W. Park (2015), "The flow of international students from a macro perspective: a network analysis", Compare: A fournal of Comparative and International Education, 7925 (April), pp. 1-27.

Beech, S. E. (2014), "Social \& Cultural Geography International student mobility: the role of social networks", Social E Cultural Geography, 9365 (May), pp. $37-41$.

Brodin, J. (2010), "Education for Global Competencies", Journal of Studies in International Education, 14(5), pp. 569584.

Brown, M. y L. Knopp (2006), "Places or polygons? Governmentality, scale, and the census in the Gay and Lesbian Atlas", Population, Space and Place, 12(4), pp. 223-242.

Castro, E. (2011), El vocabulario de Michel Foucault, Buenos Aires, Prometeo.

Cefai, S. (2014), "Introduction: cartographies of belonging: the marketisation of desire through media, practice and place", Gender, Place E Culture: A Fournal of Feminist Geography, 21(5), pp. 582-588.

Chankseliani, M. (2016). "Escaping homelands with limited employment and tertiary education opportunities: outbound studen t mobility from Post-Soviet countries", Population, Space and Place, 22(3), pp. 301-316.
Chiang, L.-C. (2012), "Trading on the western strength: the dilemmas of transnational higher education in East Asia”, Higher Education Policy, 25(7), pp. 171-189.

Collins, F. (2014), "Globalising higher education in and through urban spaces: Higher education projects, international student mobilities and trans-local connections in Seoul", Asia Pacific Viewpoint, 55(2), pp. 242-257.

Collins, F., R. Sidhu, N. Lewis y B. Yeoh (2014), "Mobility and desire: international students and Asian regionalism in aspirational Singapore", Discourse: Studies in the Cultural Politics of Education, 35(5), pp. 37-41.

Dennis, M.J. (2016), "Consider implications of Brexit for international student mobility", Enrollment Management Report, 20(6), p. 3.

Doerr, N. M. (2014), "Desired learning, disavowed learning: Scale-making practices and subverting the hierarchy of study abroad experiences", Geoforum, 54, pp. $70-79$.

Dolby, N. (2008), "Research in international education", Review of Educational Research, 78(3), pp. 676-726.

Doughty, K. y L. Murray (2016), "Discourses of mobility: institutions, everyday lives and embodiment", Mobilities, 11(2), pp. 303-322.

Douglass, J. A. (2015), "International Berkeley: past and current debates on the role of international students in an american university", Educational Studies, 1(2), pp. 76-108, <http://vo.hse.ru/data/2015/08/ 03/1085216702/2015-2 Douglas En.pdf>.

Doyle, Stephanie et al. (2016), "An investigation of factors associated with student participation in study abroad", Journal of Studies in International Education, 14, 5, pp. 471490.

Findlay, A. M. (2011), "An assessment of supply and demand-side theorizations of international student mobility", International Migration, 49(2), pp. 162-190.

Findlay, A. M., R. King, F. M. Smith, A. Geddes y R. Skeldon (2012), "World class? An investigation of globalisation, difference and international student 
mobility", Transactions of the Institute of British Geographers, 37 (1), pp. 118-131.

Forsey, M., S. Broomhall y J. Davis (2012), "Broadening the mind? Australian student reflections on the experience of overseas study", Gournal of Studies in International Education, 16(2), pp. 128-139.

Furukawa, T., N. Shirakawa y K. Okuwada (2013), "An empirical study of graduate student mobility underpinning research universities", Higher Education, 66(1), pp. 17-37.

Gao, X. (2014), “'Floating elites': interpreting mainland Chinese undergraduates' graduation plans in Hong Kong", Asia Pacific Education Review, 15(2), pp. 223-235.

Geddie, K. (2015), "Policy mobilities in the race for talent: Competitive state strategies in international student mobility", Transactions of the Institute of British Geographers, 40(2), pp. 235-248.

Gong, X. y T. Huybers (2015), "Chinese students and higher education destinations: findings from a choice experiment", Australian Fournal of Education, 0, pp. 1-23.

Healey, N. y L. Michael (2014), "Towards a new framework for analysing transnational education", Higher Education Policy, 28(3), pp. 369-391.

Higgins, R. M. y A. Brady (2016), "Language policy, planning, and enactment: the necessity and empowering potential at the local level", Current Issues in Language Planning, 17(3-4), pp. 242-259.

Kaiser, R. y E. Nikiforova (2008), "The performativity of scale: the social construction of scale effects in Narva, Estonia", Environment and Planning D: Society and Space, 26(3), pp. 537-562.

Killick, D. (2011), "Journal of Studies in International Education seeing-ourselves-in-the-world: developing global citizenship through international mobility and campus community", Journal of Studies in International Education, 20(10), pp. 1-18.

Kim, J. (2016), "Global cultural capital and global positional competition: international graduate students' transnational occupational trajectories", British fournal of Sociology of Education, 37(1), pp. 30-50.

King, R., P. Raghuram y M. Keynes (2013), "International student migration: mapping the field and new research agendas", Population, Space and Place, 137 (November 2012), pp. 127-137.

Kirkegaard, A. M. Ø. y S. M.-L. W. Nat-George (2016), "Fleeing through the globalised education system: the role of violence and conflict in international student migration", Globalisation, Societies and Education, 14(3), pp. 390-402.

Koro-Ljungberg, M. (2015), Reconceptualizing qualitative research: methodologies without methodology, Londres, SAGE Publications.

Kritz, M. M. (2015), "Why do countries differ in their rates of outbound student mobility?", Journal of Studies in International Education, pp. 1-19.

Lee, J. J. y C. Sehoole (2015), "Regional, continental, and global mobility to an emerging economy: the case of South Africa", Higher Education, 70(5), pp. 827-843.

Li, Z. y J. Lowe (2016), "Mobile student to mobile worker: the role of universities in the "war for talent", British Journal of Sociology of Education, 37(1), pp. 11-29.

Lo-Philip, S. W. Y., C. Carroll, T. L. Tan, O. Y. Ann y S. H. Seow (2015), "Transforming educational practices: Cultural learning for short-term sojourners", International Journal of Intercultural Relations, 49, pp. 223-234.

Lyotard, J.-F. (1984), The postmodern condition: a report on knowledge, Minneapolis, University of Minnesota.

Maringe, F. y N. Sing (2014), "Teaching large classes in an increasingly internationalising higher education environment: Pedagogical, quality and equity issues", Higher Education, 67(6), pp. 761-782.

Massey, D. (2008), Pelo espaço: uma nova política da espacialidade, Rio de Janeiro, Bertrand Brasil.

Massey, D. (2013), Space, place and gender, Minneapolis, University of Minnesota Press.

Matus, C. (2016), Imagining time and space in universities, Basingstoke, Palgrave-McMillan.

Nelson, L. (1999), "Bodies (and spaces) do matter: the 
limits of performativity", Gender, Place and Culture: A Journal of Feminist Geography, 6(4), pp. 331-353.

Pasztor, A. (2015), "Careers on the move: international doctoral students at an elite british university", Population, Space and Place, 21(8), pp. 832-842.

Perkins, R. y E. Neumayer (2014), "Geographies of educational mobilities: exploring the uneven flows of international students", Geographical fournal, 180(3), pp. 246-259.

Pherali, T. J. (2012), "Academic mobility, language, and cultural capital: the experience of transnational academics in British higher education institutions", Journal of Studies in International Education, 16(4), pp. 313333.

Power, S. y D. Frandji (2010), "Education markets, the new politics of recognition and the increasing fatalism towards inequality", Fournal of Education Policy, 25(3), pp. 385-396.

Rizvi, F. (2011), "Theorizing student mobility in an era of globalization", Teachers and Teaching: Theory and Practice, 17(6), pp. 693-701.

Rosière, S. (2015), "Mundialização e teicopolíticas: análise do fechamento contemporâneo das fronteiras internacionais", Boletim Gaúcho de Geografia, 42(2).

Salisbury, M. H., M. B. Paulsen y E.T. Pascarella (2011), "Why do all the study abroad students look alike? Applying an integrated student choice model to explore differences in the factors that influence white and minority students' intent to study abroad", Research in Higher Education, 52(2), pp. 123-150.

Santos, M. (2000), Por uma outra globalização, Rio de Janeiro, Record.

Schartner, A. (2016), "The effect of study abroad on intercultural competence: a longitudinal case study of international postgraduate students at a British university", fournal of Multilingual and Multicultural Development, 37(4), pp. 402-418.

Shields, R. (2014a), "Globalization and international student mobility: a network analysis", Comparative Education Review, 57 4, pp. 609-636.

Shields, R. (2014b), "Reconsidering regionalisation in global higher education: student mobility spaces of the European Higher Education Area", Compare: A Journal of Comparative and International Education, 7925(12), pp. 1-19.

Sibomana, E. (2016), “We know what to say, we know what to write, but we don't know how': the challenges of becoming academically literate in a new linguistic and socio-cultural space", Education as Change, 20(2), pp. 123-144.

Sidhu, R., F. Collins, N. Lewis y B. Yeoh (2016), "Governmental assemblages of internationalising universities: Mediating circulation and containment in East Asia", Environment and Planning A, (24).

Sidhu, R. K. y G. Dall'Alba (2012), "International education and (dis) embodied cosmopolitanisms", Educational Philosophy and Theory, 44(4), pp. 413-431.

Soria, K. M. y J. Troisi (2014), "Internationalization at home alternatives to study abroad: implications for students' development of global, international, and intercultural competencies", Fournal of Studies in International Education, 18(3), pp. 261-280.

Sterzuk, A. (2014), “The standard remains the same': language standardisation, race and othering in higher education", Fournal of Multilingual and Multicultural Development, 4632 (March), pp. 1-14.

Tannock, S. (2013), "When the demand for educational equality stops at the border: wealthy students, international students and the restructuring of higher education in the UK", fournal of Education Policy, 28(4), pp. $449-464$.

UNESCO (2016), "Global flow of tertiary-level students", en <http://www.uis.unesco.org/Education/Pages/ international-student-flow-viz.aspx $>$.

Van Oorschot, I. (2013), "Negotiating knowledges abroad: non-Western students and the global mobility of knowledge", Compare: A Journal of Comparative and International Education, 7925 (August 2014), pp. 1-21.

Walker, P. (2013), "International student policies in UK higher education from colonialism to the coalition: developments and consequences", fournal of Studies in International Education, 18(4), pp. 325-344. 
Waters, J. L. (2016), "Education unbound? Enlivening debates with a mobilities perspective on learning", Progress in Human Geography, (Online First), pp. 1-20.

Waters, J. L. y M. W. H. Leung (2016), "Domesticating transnational education: discourses of social value, self-worth and the institutionalisation of failure in 'meritocratic", Transactions of the Institute of British Geographers, Hong Kong.

Weiss, M. L. y M. Ford (2011), "Temporary transnationals: Southeast Asian students in Australia", fournal of Contemporary Asia, 41 (March 2015), pp. 229-248.

Wynveen, C. J., G. T. Kyle y M. A. Tarrant (2012), "Study abroad experiences and global citizenship: fostering proenvironmental behavior", fournal of Studies in International Education, 16(4), pp. 334-352.

Ye, R. (2015), "Transnational higher education strategies into and out of Singapore: commodification and consecration", TRaNS: Trans-Regional and -National Studies of Southeast Asia, 4(1), pp. 85-108.

Ziguras, C. y A. T. N. Pham (2014), "Assessing participation in cross-border higher education in cities: Foreign education provision in Ho Chi Minh City", Asia Pacific Viewpoint, 55(2), pp. 169-181.. 